\title{
Rib waveguides for mid-infrared silicon photonics
}

\author{
Milan M. Milošević, ${ }^{1, *}$ Petar S. Matavulj, ${ }^{2}$ Pengyuan Y. Yang, ${ }^{1}$ Alvise Bagolini, ${ }^{3}$ and Goran Z. Mashanovich ${ }^{1}$ \\ ${ }^{1}$ Advanced Technology Institute, Faculty of Engineering and Physical Sciences, University of Surrey, Guildford, \\ Surrey GU2 7XH, United Kingdom \\ ${ }^{2}$ Faculty of Electrical Engineering, University of Belgrade, Bulevar kralja Aleksandra 73, 11120 Belgrade, Serbia \\ ${ }^{3}$ B. Kessler Foundation for Research, Via Sommarive 18, Povo, 38100 Trento, Italy \\ *Corresponding author: m.milosevic@surrey.ac.uk
}

Received April 13, 2009; revised July 6, 2009; accepted July 20, 2009;

posted July 23, 2009 (Doc. ID 110077); published August 20, 2009

\begin{abstract}
Design rules for both single-mode and polarization-independent strained silicon-on-insulator rib waveguides at the wavelength of $3.39 \mu \mathrm{m}$ are presented for the first time to our knowledge. Waveguide geometries with different parameters, such as waveguide height, rib width, etch depth, top oxide cover thickness and sidewall angle, have been studied in order to investigate and define design rules that will make devices suitable for mid-IR applications. Chebyshev bivariate interpolation with a standard deviation of less than $1 \%$ has been used to represent the zero-birefringence surface. Experimental results for the upper cladding stress level have been used to determine the influence of top oxide cover thickness and different levels of upper cladding stress on waveguide characteristics. Finally, the polarization-insensitive and single-mode locus is presented for different waveguide heights. (C) 2009 Optical Society of America
\end{abstract}

OCIS codes: $130.0130,250.0250,230.7370,260.3060,260.5430$.

\section{INTRODUCTION}

The mid-IR spectral region is very interesting, as the practical realization of optoelectronics devices operating in this wavelength range offers potential applications in a wide variety of areas, including optical sensing and environmental monitoring, free-space communications, biomedical and thermal imaging, and IR countermeasures. Many pollutant and toxic gases and liquids that we wish to detect or monitor exhibit bands of absorption lines in the IR part of the spectrum. Consequently, the mid-IR is very attractive for the development of sensitive optical sensor instrumentation. In addition, there are two atmospheric transmission windows $(3-5$ and $8-14 \mu \mathrm{m})$ that permit free-space optical communications. Of interest are also thermal imaging applications in both civil and military situations. Mid-IR photonics also offers the potential for development of minimally invasive, effective, and safe diagnostic techniques. This spectral range is attractive for highly precise surgical procedures and medical ablation of tissue because of its high absorption in water, and hence small penetration depths, especially for wavelengths around $3 \mu \mathrm{m}$, where the penetration depth can be as small as a few micrometers [1]. However, the advantages of this wavelength range have not been fully exploited owing to the limitations in current technology. The potential of this wavelength range is being pursued, via research in the mid-IR sources and detectors based on type III-V materials, particularly in quantum cascade lasers and detectors [2,3] and quantum well and quantum dot lasers and detectors [4]. Nevertheless, relatively little results have been published on mid-IR silicon photonics. Silicon has two low-loss transmission windows, one from 1.2 to $8 \mu \mathrm{m}$ and the other from 24 to $100 \mu \mathrm{m}$ [5]. However, $\mathrm{SiO}_{2}$ is very lossy in the $2.6-2.9 \mu \mathrm{m}$ range and be- yond $3.6 \mu \mathrm{m}$ [6]. Soref et al., proposed several waveguide structures suitable for mid- and long-IR spectral regions [7]. In this paper we theoretically analyze conventional rib waveguides for mid-IR applications for what is the first time to our knowledge.

The structure we analyze in this paper, at the operating wavelength of $\lambda=3.39 \mu \mathrm{m}$ is a silicon-on-insulator (SOI) rib waveguide with the height $H \in(2.50$, $2.95,3.50) \mu \mathrm{m}$, and variable waveguide rib width $W$, etch depth $D$, top oxide cover thickness $t$, and sidewall angle $\theta$, as shown in Fig. 1. This wavelength was chosen in the design stage because it is interesting for sensing applications; a Raman amplification was also recently reported at this wavelength [8], and it falls within the first atmospheric transmission window $(3-5 \mu \mathrm{m})$, which is interesting for free-space communications. The rib waveguide height of $H=2.95 \mu \mathrm{m}$ has been chosen, as it scales up with the height of $H=1.35 \mu \mathrm{m}$ of the rib waveguides used in the optical filters reported by Headley et al. at the wavelength of $1.55 \mu \mathrm{m}$ [9]. The buried oxide layer is $1 \mu \mathrm{m}$ in thickness, while the top oxide cladding in the simulations performed is $0.1-3 \mu \mathrm{m}$ thick. The strain in the upper $\mathrm{SiO}_{2}$ layer produces a stress distribution within and near the silicon rib, which in turn causes a change of the refractive index in both materials due to the photoelastic effect [10]. During the simulations, we used that the thickness of the upper cladding film on the rib sidewalls is $70 \%$ of that on the top, a result obtained by scanning electron microscope investigation of the fabricated rib waveguides (Fig. 1, inset). The simulations were performed by using $2 \mathrm{D}$ finite element method modeling [11-13] and verified by a 3D semivectorial and fullvectorial beam propagation method [14]. The method we used was to calculate the effective indexes for the funda- 


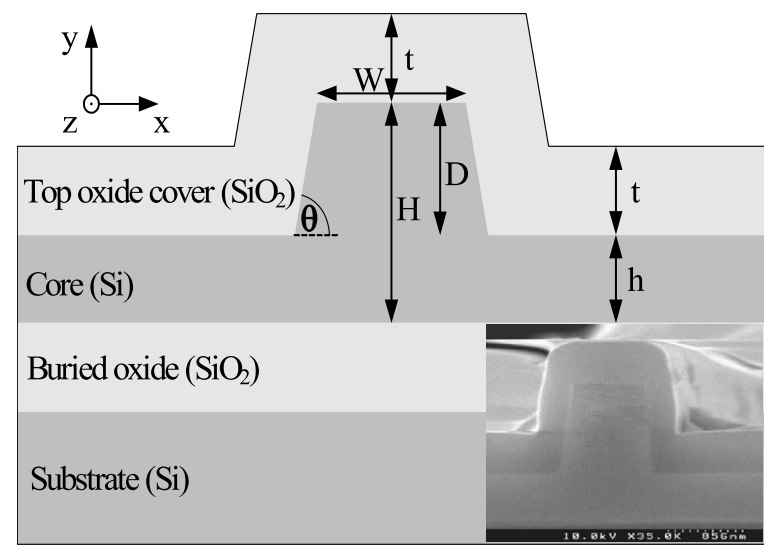

Fig. 1. Modeled structure: $t$ is the top oxide layer thickness, $H$ is rib height, $D(D=H-h)$ is etch depth, $W$ is waveguide rib width, and $\theta$ is the rib sidewall angle. Inset, scanning electron microscope structure.

mental and the first two higher-order modes, for both polarizations, TE (transverse electric) and TM (transverse magnetic), over a range of waveguide dimensions.

The paper is organized as follows. In Section 2 the stress theory is described and the experimental results for top oxide cladding stress are presented. The stress influence on the total birefringence for different waveguide geometries is presented in Section 3. The polarizationindependent surface and the influence of the waveguide sidewall angle is also explained. Experimental values for cladding stress have been used to describe the influence of different values of stress on waveguide birefringence. The investigation of single-mode behavior and satisfying both single-mode and polarization-independence conditions for various waveguide heights is presented in Section 4. The concluding remarks are given in Section 5.

\section{TOP OXIDE CLADDING STRESS}

The change in the material effective indexes caused by stress-induced effects can be expressed as [15]

$$
\begin{aligned}
& \Delta N_{x}=N_{x}-N_{0}=-C_{1} \sigma_{x}-C_{2}\left(\sigma_{y}+\sigma_{z}\right), \\
& \Delta N_{y}=N_{y}-N_{0}=-C_{1} \sigma_{y}-C_{2}\left(\sigma_{x}+\sigma_{z}\right),
\end{aligned}
$$

where $\sigma_{x}, \sigma_{y}$, and $\sigma_{z}$ are the principal components of the relative stress tensor, $N_{x}$ and $N_{y}$ are the components of the material's refractive index, $N_{0}$ is the refractive index without stress, and $C_{1}$ and $C_{2}$ are the stress-optic constants related to the Young's modulus $(E)$, Poisson's ratio $(\nu)$, and the photoelastic tensor elements $\left(p_{11}\right.$ and $\left.p_{12}\right)$ as

$$
\left(\begin{array}{l}
C_{1} \\
C_{2}
\end{array}\right)=\frac{N_{0}^{3}}{2 E}\left(\begin{array}{cc}
1 & -2 \nu \\
-\nu & 1-\nu
\end{array}\right)\left(\begin{array}{l}
p_{11} \\
p_{12}
\end{array}\right) .
$$

One source of birefringence is strain due to the temperature difference before and after fabrication processes. The strain and stress are related as follows $[16,17]$ :

$$
\left(\begin{array}{c}
\varepsilon_{x} \\
\varepsilon_{y} \\
\varepsilon_{z}
\end{array}\right)=\frac{1}{E}\left(\begin{array}{ccc}
1 & -\nu & -\nu \\
-\nu & 1 & -\nu \\
-\nu & -\nu & 1
\end{array}\right)\left(\begin{array}{c}
\sigma_{x} \\
\sigma_{y} \\
\sigma_{z}
\end{array}\right)+\left(\begin{array}{c}
\alpha \Delta T \\
\alpha \Delta T \\
\alpha \Delta T
\end{array}\right),
$$

where $\varepsilon_{x}, \varepsilon_{y}$, and $\varepsilon_{z}$ are the principal strain components along the $x, y$ and $z$ directions, respectively, $\alpha$ is the thermal expansion coefficient, and $\Delta T=T_{0}-T_{\text {ref }}$ represents the difference between the operating temperature $T_{0}$ and reference temperature $T_{\text {ref. }}$. At the reference temperature, which corresponds to the deposition temperature for an idealized oxide film on a silicon system, there is no intrinsic stress in any layer, and all the material layers are at equilibrium. As can be seen from Eq. (3), the strain consists of the photoelastic stress and thermally induced stress. By inverting Eq. (3) the relation for the stress distribution tensors can be obtained as

$$
\begin{aligned}
\left(\begin{array}{c}
\sigma_{x} \\
\sigma_{y} \\
\sigma_{z}
\end{array}\right)= & \frac{E}{(1+\nu)(1-2 \nu)}\left(\begin{array}{ccc}
1-\nu & \nu & \nu \\
\nu & 1-\nu & \nu \\
\nu & \nu & 1-\nu
\end{array}\right)\left(\begin{array}{l}
\varepsilon_{x} \\
\varepsilon_{y} \\
\varepsilon_{z}
\end{array}\right) \\
& -\frac{\alpha E \Delta T}{1-2 \nu}\left(\begin{array}{l}
1 \\
1 \\
1
\end{array}\right) .
\end{aligned}
$$

All material parameters used in numerical calculations were set to be equal to those used in [10]. The calculation window size was chosen to be large enough to minimize the influence of the edge effects on the stress distribution in the vicinity of the rib waveguide. Because of the sharp waveguide corners, stress is inhomogeneous and anisotropic [18]. The stress field in the waveguide and Maxwell's equations are solved by the finite element method, on a nonuniform mesh of triangular elements [11]. For our numerical computations, approximately 40000 elements and the higher-order shape functions of the Lagrange type were used.

We have measured the stress, and Table 1 summarizes experimental results for upper cladding stress for a certain value of deposited oxide thickness. Silicon oxide layers were deposited on $500 \mu \mathrm{m}$ thick silicon wafers, oriented along the $\langle 100\rangle$ plane. Thermal silicon oxide was grown in a standard $4 \mathrm{in}$. furnace at a temperature of $1150^{\circ} \mathrm{C}$. Plasma-enhanced chemical vapor deposition (PECVD) silicon oxide was deposited by using a STS (Surface Technology Systems, Ltd.) coupled planar parallel electrode system. The temperature of the wafer chuck during deposition was $300^{\circ} \mathrm{C}$. The plasma was generated

Table 1. Experimental Results for Cladding Stress Measurements and Corresponding Standard Deviation of Measured Data ${ }^{a}$

\begin{tabular}{ccc}
\hline Layer & Stress $(\mathrm{MPa})$ & Thickness $(\mathrm{nm})$ \\
\hline $\mathrm{SiO}_{2}$ (thermal) & $-378( \pm 6)$ & 270 \\
$\mathrm{SiO}_{2}$ (thermal) & $-344( \pm 2)$ & 470 \\
$\mathrm{SiO}_{2}$ (thermal) & $-324( \pm 1)$ & 1000 \\
PECVD SiO (high frequency) & $-253( \pm 2)$ & 1110 \\
PECVD SiO (low frequency) & $-388( \pm 1)$ & 1150 \\
\hline
\end{tabular}

${ }^{a}$ Experimentally measured $\sigma_{\text {film }}$ was used as a target stress level in the simulation analysis. 
by using a mixture of $\mathrm{N}_{2}, \mathrm{~N}_{2} \mathrm{O}$, and $\mathrm{SiH}_{4}$ gases. Two sets of power and frequency were used for the deposition of silicon oxide, $60 \mathrm{~W}$ at $308 \mathrm{KHz}$ (low-frequency PECVD) and $30 \mathrm{~W}$ at $13.56 \mathrm{MHz}$ (high-frequency PECVD). Stress measurement was carried out by wafer curvature comparison, before and after deposition, by using Hoffmann's formula for thin films [19]:

$$
\sigma_{\text {film }}=\frac{E_{s} h_{s}}{6\left(1-\nu_{s}\right) R t_{d}},
$$

where $E_{s}$ is the Young's modulus of the substrate, $\nu_{s}$ is Poisson's ratio of the substrate, $E_{s} /\left(1-\nu_{s}\right)$ is the biaxial elastic modulus of the substrate, $R$ is the effective wafer radius of curvature, $h_{s}$ is the thickness of the substrate, $t_{d}$ is the thickness of the deposited layer and $\sigma_{\text {film }}$ is the average film stress. For a single-crystal silicon wafer, $E_{s}$ was assumed to be equal to $155.8 \mathrm{GPa}$, while $\nu_{s}$ is equal to 0.2152 . The effective radius of curvature $R$ is calculated as $R=R_{1} R_{2} /\left(R_{1}-R_{2}\right)$, where $R_{1}$ and $R_{2}$ are the bow radii of the wafer before and after the film deposition. All wafers used were oriented along the $\langle 100\rangle$ plane, while the profile measurements followed the $\langle 100\rangle$ direction. Wafer curvature was measured by a KLA-Tencor profilometer, with dedicated patterning of the underlying silicon substrate to assure perfect overlapping of scanning paths before and after deposition. The experimentally measured $\sigma_{\text {film }}$ (Table 1 ) was used as a target stress level in the simulation analysis by varying the $\Delta T$ in the model [10].

Figure 2 represents an example of calculated stress field distribution in the waveguide and corresponding electric field profiles. The cladding film in-plane $(x-z$ plane) stress component $\sigma_{\text {film }}$ represents the stress in the uniform film far from the rib. By changing $\Delta T$ in Eq. (4) we modeled experimentally measured stress from Table 1. As can be seen, stress is very anisotropic in the waveguide core and is governed mainly by the upper cladding. The electric field is concentrated in the waveguide core and decays rapidly in the top oxide cladding and buried oxide layer. The increased polarization dependence in the waveguide is derived from the increasingly differing mode shapes of the quasi-TE and quasi-TM modes.
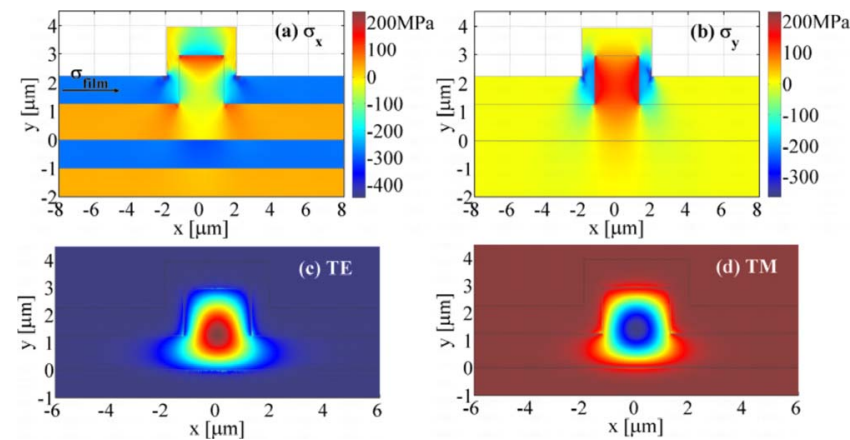

Fig. 2. (Color online) Stress field distribution in a SOI rib waveguide. (a) Stress field $\sigma_{x}$, (b) stress field $\sigma_{y}$ and corresponding electric field profiles: (c) quasi-TE mode, (d) quasi-TM mode. $H$ $=2.95 \mu \mathrm{m}, \quad D=1.7 \mu \mathrm{m}, \quad W=2.5 \mu \mathrm{m}, \quad t=1 \mu \mathrm{m}, \quad \theta=90^{\circ}, \quad \sigma_{\text {film }}$ $=-290 \mathrm{MPa}, \lambda=3.39 \mu \mathrm{m}$.

\section{POLARIZATION-INDEPENDENT RIB WAVEGUIDES}

The stress causes birefringence [20], which may in turn induce polarization conversion and polarizationdependent loss. In many applications it is important to achieve small modal birefringence, defined here as the difference between the effective indexes of the two orthogonally polarized modes, quasi-TE and quasi-TM $\Delta N_{\mathrm{eff}}=N_{\mathrm{eff}}^{\mathrm{TE}}-N_{\mathrm{eff}}^{\mathrm{TM}}$. To achieve zero birefringence in rib waveguides, an optimization of waveguide dimensions is necessary. As we are investigating a most common situation where there is an oxide upper cladding, the total birefringence is, however, the sum of the geometrical birefringence and stress-induced birefringence [10].

The modal birefringence $\Delta N_{\text {eff }}$ was evaluated for different rib waveguide geometries and top oxide cover thicknesses. Etch depth was varied for a certain $t$ and parabolas that correspond to waveguide dimensions with no polarization dependence $\left(\Delta N_{\text {eff }}=0\right)$, i.e., the zerobirefringence condition (ZBC), were plotted.

The ZBC curves for different values of top oxide cover thickness as a function of waveguide rib width and etch depth are shown in Fig. 3. It can be noticed that the increase of the top oxide cover thickness shifts the ZBC curves to smaller values of the etch depth. Also, for the upper part of the ZBC curves, that is, for wider ribs, when the oxide thickness increases, the birefringence will become negative; therefore, to make it zero again, the width of the rib needs to be increased to reduce the stress's influence on the rib. On the other hand, for the bottom part of the ZBC curves, that is, for narrower ribs, the mode resides mainly in the slab region, and therefore the increase in the oxide thickness will not affect the birefringence significantly, leading to the saturation in that region. It can be also noticed that there is no significant difference between ZBC curves for $t>2 \mu \mathrm{m}$.

If we now calculate $\mathrm{ZBC}$ points for each value of upper oxide thickness for each etch depth and corresponding waveguide rib width value, it is possible to plot the ZBC

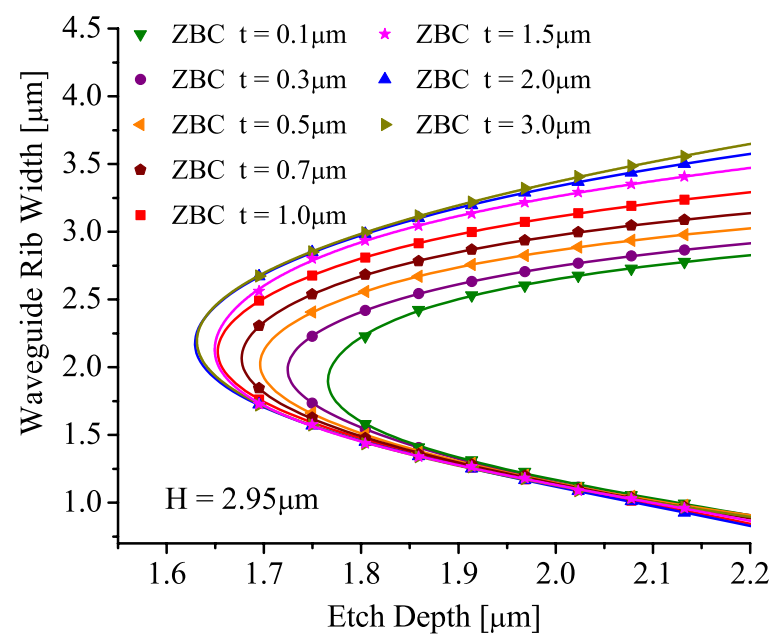

Fig. 3. (Color online) ZBC as a function of waveguide rib width and etch depth for different values of top oxide thickness at an operating wavelength of $\lambda=3.39 \mu \mathrm{m}$. The waveguide height is equal to $H=2.95 \mu \mathrm{m}$, while the upper cladding stress is equal to $\sigma_{\text {film }}=-290 \mathrm{MPa}\left(\theta=90^{\circ}\right)$. 
surface (Fig. 4). This surface can be interpolated by using Chebyshev bivariate interpolation of the fourth-order polynomial [Eq. (6)],

$$
\begin{aligned}
D= & a+b T_{1}(t)+c T_{1}(W)+d T_{2}(t)+e T_{1}(t) T_{1}(W)+f T_{2}(W) \\
& +g T_{3}(t)+h T_{2}(t) T_{1}(W)+i T_{1}(t) T_{2}(W)+j T_{3}(W)+k T_{4}(t) \\
& +l T_{3}(t) T_{1}(W)+m T_{2}(t) T_{2}(W)+n T_{1}(t) T_{3}(W)+o T_{4}(W)
\end{aligned}
$$

where $T_{n}(x)$ represents the Chebyshev polynomial of the first kind defined by the contour integral [21]:

$$
T_{n}(x)=\frac{1}{4 \pi i} \oint \frac{\left(1-t^{2}\right) t^{-n-1}}{1-2 t x+t^{2}} \mathrm{~d} t,
$$

where the contour encloses the origin and is traversed in a counterclockwise direction. This polynomial has been chosen because it yields a minimal standard error $(<1 \%)$ compared with other types of interpolation such as sigmoid bivariate series of the sixth order, cosine bivariate series of the fifth order, and Fourier bivariate models [22]. The coefficients in Eq. (6) are 2.20635966, $-0.2768732,0.318018557,0.077150967,-0.40688008$, $0.545797812,-0.00778914, \quad 0.10441509,-0.21887309$, $0.065419062,-0.00544293,-0.00574398,0.042804536$, $-0.06152694,0.022109798$, from $a$ to $o$, respectively, while waveguide parameters in Eq. (6) are in micrometers.

The results show that for smaller etch depths ( $D$ $<D_{\min }=1.6 \mu \mathrm{m}$ ) the birefringence is always positive, i.e., the effective refractive indices for both polarizations cannot be equalized by changing the values of waveguide width and top oxide cover. It should be noticed that these results are obtained for a certain level of stress of the upper oxide cladding and constant waveguide height; hence $D_{\text {min }}$ will be different for different levels of stress and height. Deeper etched devices show a tendency for two specific waveguide widths for which the ZBC is fulfilled. The increase of the top oxide cover thickness will allow the presence of shallower etch depths at which both modes will be guided through the waveguide in the same fashion. For larger $t$, the family of ZBC curves becomes deeper and wider. Considering constant etch depth, when

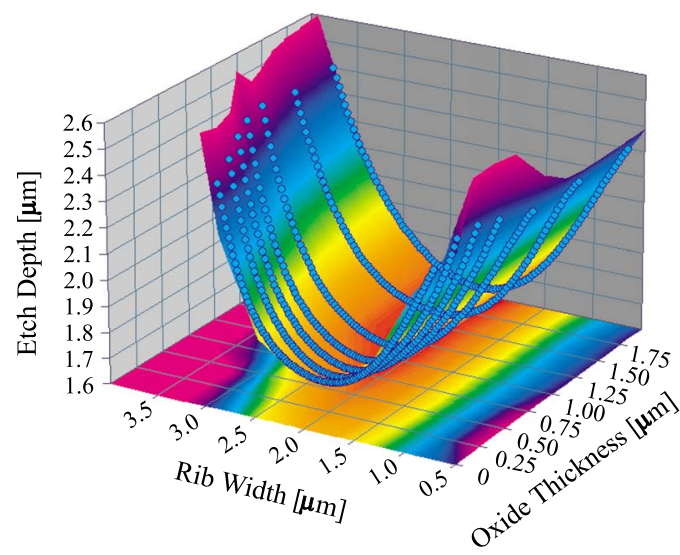

Fig. 4. (Color online) Zero-birefringent surface for an operating wavelength of $\lambda=3.39 \mu \mathrm{m}$, waveguide height of $H=2.95 \mu \mathrm{m}$ and cladding stress of $\sigma_{\text {film }}=-290 \mathrm{MPa}\left(\theta=90^{\circ}\right)$. $t$ is increased, the larger value of $W$ for which ZBC is fulfilled will also increase, while the lower value of $W$ will not change significantly and will be saturated, as already shown in Fig. 3.

Figure 5 shows the influence of sidewall angle on the ZBC. The ZBC curve is shifted to lower values of $W$. When $\theta$ is decreased, for constant $W$, the width of the bottom of the rib will increase, and therefore the effective width of the rib will increase. Therefore, for constant oxide thickness, the ZBC will not be fulfilled. To obtain $\Delta N_{\text {eff }}=0$, the effective rib width needs to be decreased, which means that the ZBC will be fulfilled for narrower ribs. Fabricated waveguides typically have sidewall angles in the $80^{\circ}-90^{\circ}$ range [23]. In that case the $\mathrm{ZBC}$ curve moves down, maintaining the values of etch depth (Fig. 5). Equation (8) describes the influence of the sidewall angle on the waveguide width for maintaining the $\mathrm{ZBC}$ for $80^{\circ} \leqslant \theta \leqslant 90^{\circ}, \quad D=1.65 \mu \mathrm{m}, \quad \lambda \in\{2.9,3.6\} \mu \mathrm{m}$ and $\sigma_{\text {film }}$ $=-290 \mathrm{MPa}$ :

$$
W[\mu \mathrm{m}]=(-0.3058+0.6142 \cdot \theta[\mathrm{rad}]) \cdot \lambda[\mu \mathrm{m}] .
$$

This equation is complementary to the previous, and together they give conditions for waveguide parameters that have to be matched to satisfy the ZBC.

The influence of different waveguide heights on the ZBC for different values of top oxide cover thickness is presented in Fig. 6. The curves follow a similar trend compared with the previous case for the waveguide height of $2.95 \mu \mathrm{m}$ : each curve shifts to the right, for relatively smaller top oxide cover thicknesses, and saturates for small values of $W$, owing to the optical mode confinement in the slab region. If we look a family of $\mathrm{ZBC}$ curves for $H=2.95 \mu \mathrm{m}$ and consider it as a starting point, it can be seen that for waveguide height of $H=2.5 \mu \mathrm{m}$ the family of ZBC curves shifts to the left, providing a minimum value of etch depth of $D_{\min }=1.4 \mu \mathrm{m}$ for which the ZBC can be achieved. Similarly, the family of ZBC curves for $H$ $=3.5 \mu \mathrm{m}$ shifts to the right and gives $D_{\min }=1.85 \mu \mathrm{m}$. This means that by decreasing the waveguide height the ZBC can be achieved for shallower etched devices.

It is interesting to see what happens when the influence of various cladding stress levels are taken into account (Fig. 7) for a specific waveguide geometry. Here, for a waveguide height of $H=2.95 \mu \mathrm{m}$ and top oxide cover of $t=1 \mu \mathrm{m}$, four examples are presented, $\sigma_{\text {film }} \in(-250$,

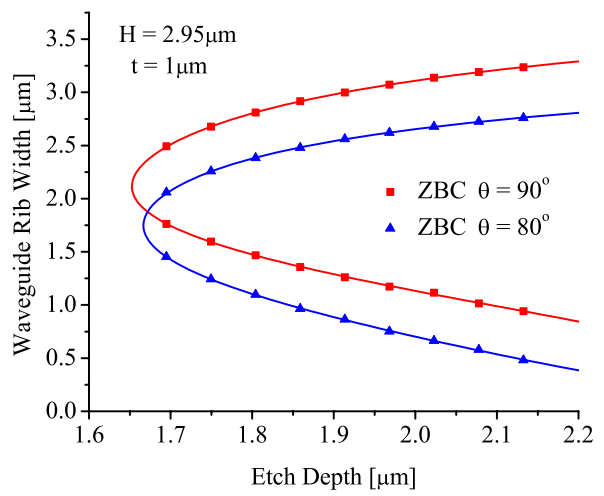

Fig. 5. (Color online) ZBC as a function of waveguide rib width and etch depth for different values of rib sidewall angle. $\lambda$ $=3.39 \mu \mathrm{m}, H=2.95 \mu \mathrm{m}, t=1 \mu \mathrm{m}, \sigma_{\text {film }}=-290 \mathrm{MPa}$. 

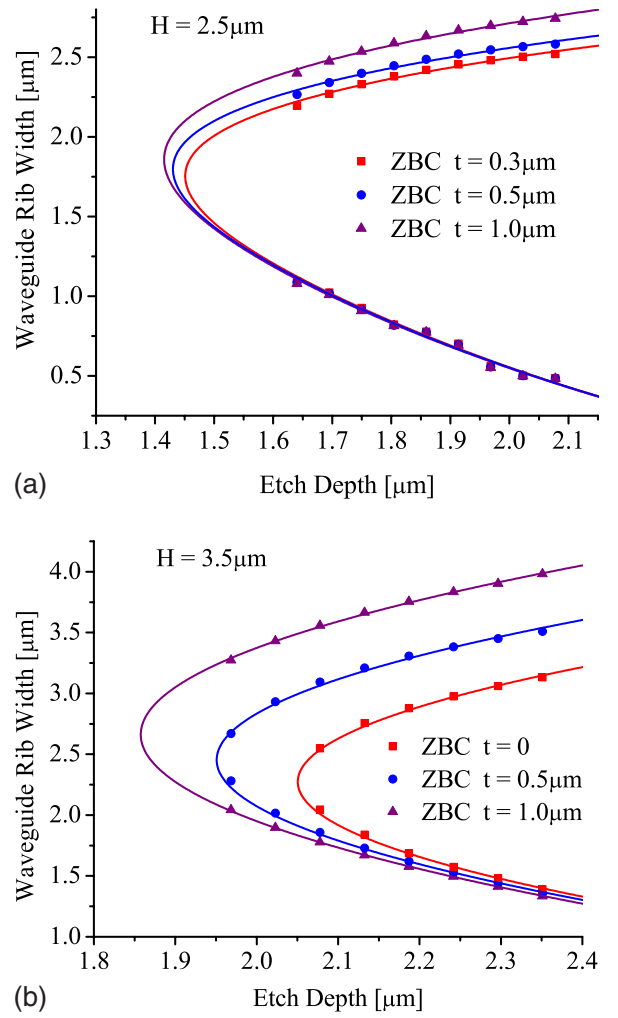

Fig. 6. (Color online) ZBC as a function of waveguide rib width and etch depth for different values of top oxide thickness for waveguide height of (a) $H=2.5 \mu \mathrm{m}$ and (b) $H=3.5 \mu \mathrm{m} . \lambda$ $=3.39 \mu \mathrm{m}, \theta=90^{\circ}, \sigma_{\text {film }}=-290 \mathrm{MPa}$.

-290, -350, -400) $\mathrm{MPa}$, using the previously shown experimental data. It can be seen that the ZBC curve shifts to lower values of $D$ when higher value of compressive stress is applied. For small values of $W$ (narrower widths), the mode is guided mainly through the slab region, and an increase in compressive cladding stress will not significantly affect the birefringence, providing saturation of ZBC curves in this region. As $\sigma_{\text {film }}$ represents the in-plane $\left(\sigma_{x}\right)$ compressive stress, for larger values of $W$ the waveguide is effectively compressed in the $x$ direction, providing a smaller value for effective waveguide width and causing birefringence. In order to achieve the $\mathrm{ZBC}$, the effective waveguide width needs to be increased to balance the influence of compression in plain strain, and

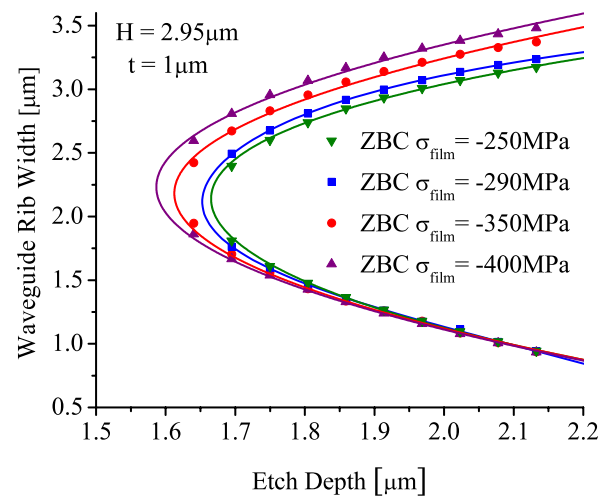

Fig. 7. (Color online) ZBC as a function of waveguide rib width and etch depth for different values of upper cladding stress levels. $\lambda=3.39 \mu \mathrm{m}, H=2.95 \mu \mathrm{m}, t=1 \mu \mathrm{m}, \theta=90^{\circ}$. that is the reason why the curves move toward higher values of $W$ when the absolute value of stress increases. This case is similar to that previously described, because a greater top oxide cover thickness $t$ corresponds to a higher absolute value of comprehensive cladding stress.

As it can be seen, a careful design of waveguides need to be carried out prior to the fabrication to achieve polarization independence.

\section{SINGLE-MODE AND POLARIZATION- INDEPENDENT WAVEGUIDES}

Similar to the calculation of the ZBC curves, for a given waveguide height and oxide thickness, a number of single-mode quasi-TE and quasi-TM points were found in the waveguide width, etch depth plane. We focused on one specific value for etch depth and changed the waveguide rib width until the first higher-order mode was found. This was done for a given value of waveguide height and top oxide layer thickness. Field profile is strongly dependent on the waveguide geometry, and in some cases we found that the more critical higher-order mode was the quasi-TM $\mathrm{TM}_{01}$ mode, while in other cases it was the quasi- $\mathrm{TM}_{10}$. In common with our previous work [24,25], we found that satisfying the single-mode condition (SMC) for the TM mode is again more restrictive than for the TE mode. The area below the SMC for quasi-TM mode defines the global condition for the single-mode behavior. According to our simulations for given waveguide parameters, there is at least one intersection point between the SMC line for quasi-TM mode and the ZBC curve. A part of the ZBC parabola between these intersection points (locus) defines waveguide parameters for which both the ZBC and the SMC are satisfied.

Figure 8 shows the SMC together with the ZBC curve described previously for three different waveguide heights $H \in(2.50,2.95,3.50) \mu \mathrm{m}$ at the wavelength of $\lambda$ $=3.39 \mu \mathrm{m}$. For a top oxide cover of $t=1 \mu \mathrm{m}$ single-mode curves were calculated by assuming vertical rib sidewalls and a stress level of $\sigma_{\text {film }}=-290 \mathrm{MPa}$. In the previous section we described the influence of the waveguide height on waveguide birefringence. ZBC curves move to the left while the value of waveguide height increases. This provides lower values of the waveguide etch depth for which polarization independence can be achieved. Single-mode curves are also affected by the change of the waveguide height. The slope of the SMC curve becomes larger when the waveguide height decreases. As it can be seen there is at least one intersection point between the $\mathrm{ZBC}$ and $\mathrm{SMC}$ curve. The part of the ZBC parabola from this intersection point provides a ZBC locus for which both conditions can be achieved.

Figure 9 shows the single-mode and polarizationindependent locus at different waveguide heights. The locus moves to the right when the waveguide height increases. The height can be interpolated by the expression

$$
D=\frac{a+c H+e \ln W+g H^{2}+i(\ln W)^{2}+k H \ln W}{1+b H+d \ln W+f H^{2}+h(\ln W)^{2}+j H \ln W},
$$

$H \in[2.5,3.5] \mu \mathrm{m}, W \in\left[W_{\min }, W_{\max }\right]$, where $W_{\min }$ and $W_{\max }$ are given by the following equations: 

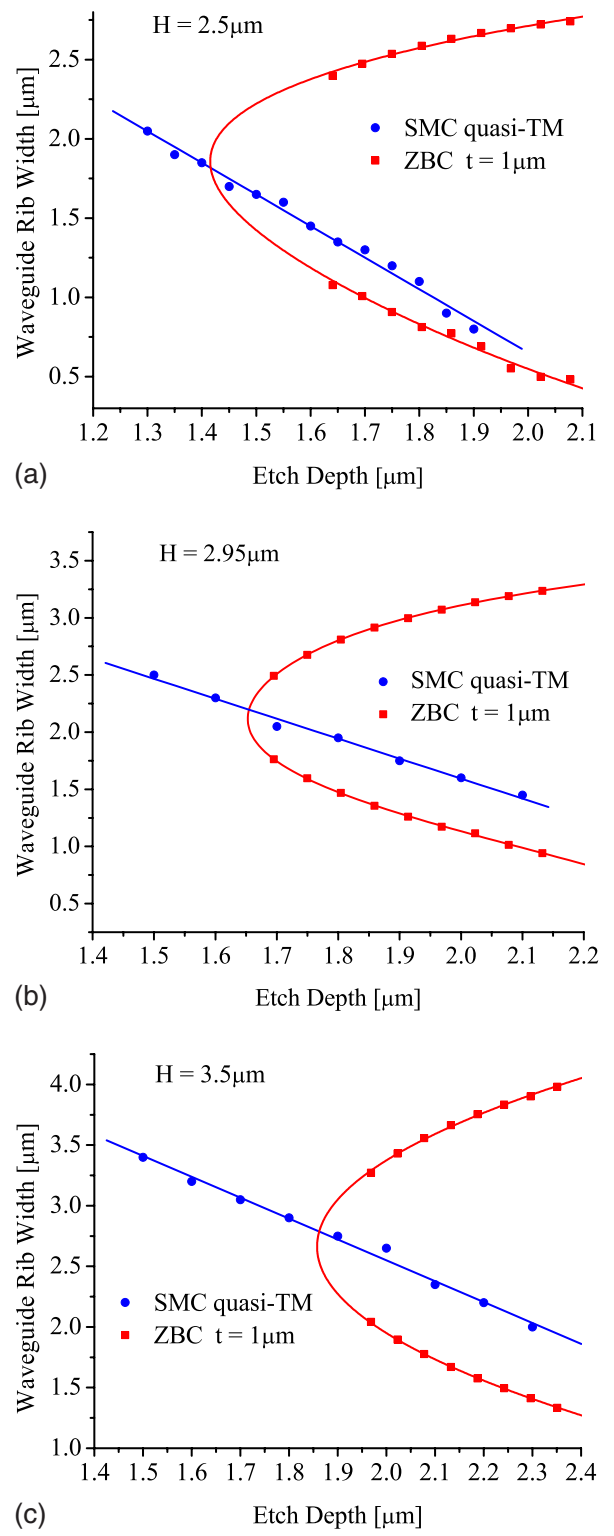

Fig. 8. (Color online) SMC and ZBC as a function of waveguide rib width and etch depth for waveguide height of (a) $H=2.5 \mu \mathrm{m}$, (b) $H=2.95 \mu \mathrm{m}$, (c) $H=3.5 \mu \mathrm{m} . \lambda=3.39 \mu \mathrm{m}, t=1 \mu \mathrm{m}, \theta=90^{\circ}$, $\sigma_{\text {film }}=-290 \mathrm{MPa}$.

$$
\begin{gathered}
W_{\min }=-1.21982+0.66379 H-0.00292 H^{2}, \\
W_{\max }=2.72265-1.23862 H+0.35506 H^{2} .
\end{gathered}
$$

This interpolation gives a minimal standard error $(\sim 0.26 \%)$, and all parameters in Eqs. (9) and (10) are in micrometers. The coefficients in Eq. (9) are 1.5363208, $-0.55421541,-0.89935048,-0.37045017,-0.50381279$, $0.085060013, \quad 0.15360476, \quad 0.059839912,0.1856229$, $0.083117058,0.057757$, from $a$ to $k$, respectively. It is worth mentioning again that this has been calculated for a relatively high value of the top oxide cover of $t=1 \mu \mathrm{m}$ and cladding stress of $\sigma_{\text {film }}=-290 \mathrm{MPa}$. As the increase of the top oxide cover to greater than $1 \mu \mathrm{m}$ will saturate the ZBC and SMC curves, the Eqs. (9) and (10) are also valid for higher values of $t(t>1 \mu \mathrm{m})$.

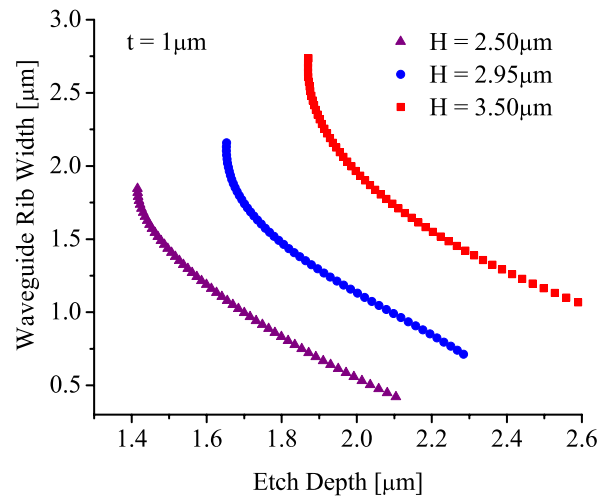

Fig. 9. (Color online) Waveguide geometries that exhibit both single-mode and polarization-independence conditions at three different waveguide heights. $\lambda=3.39 \mu \mathrm{m}, t=1 \mu \mathrm{m}, \theta=90^{\circ}, \sigma_{\text {film }}$ $=-290 \mathrm{MPa}$.

\section{CONCLUSION}

In this paper, the design rules that satisfy both singlemode and polarization-independent operation for SOI rib waveguides that can find application in the mid-IR wavelength region have been presented. We have examined waveguides with different sidewall angles, top oxide cover thicknesses, heights, rib widths, etch depths, and cladding stress levels. The measured stress value in the upper oxide cladding was used as a target stress level in numerical calculations. The stress-induced effects must not be neglected in modeling, because the zero-birefringence curves move to smaller values of etch depth and saturate for larger values of top oxide cover at a given height and cladding stress. Compressive stress shifts ZBC curves to smaller values of $D$, for constant $W$. It is interesting that for narrower ribs, the stress does not influence the ZBC significantly, as the mode resides mainly in the slab region. It has been shown that by stress engineering, zerobirefringence can be achieved for a waveguide that has otherwise relatively large birefringence. A single-mode and polarization-insensitive locus has been found at different waveguide heights determined by a simple equation that describes design rules for achieving both requirements at high values of top oxide cover. This locus can be increased by changing the sidewall angle from $90^{\circ}$ to a lower value.

\section{ACKNOWLEDGMENTS}

This work was supported in part by the Royal Society UK and the EPSRC UK Silicon Photonics programme and in part by the Serbian Ministry of Science under contract 141006A.

\section{REFERENCES}

1. A. Krier, ed., Mid-Infrared Semiconductor Optoelectronics, (Springer-Verlag, 2006).

2. M. Graf, N. Hoyler, M. Giovannini, J. Faist, and D. Hofstetter, "InP-based quantum cascade detectors in the mid-infrared," Appl. Phys. Lett. 88, 241118 (2006).

3. Q. Yang, C. Manz, W. Bronner, K. Köhler, and J. Wagner, "Room-temperature short-wavelength $(\lambda \sim 3.7-3.9 \mu \mathrm{m})$ GaInAs/AlAsSb quantum-cascade lasers," Appl. Phys. Lett. 88, 121127 (2006). 
4. M. Razeghi, C. Miesner, O. Röthig, K. Brunner, and G. Abstreiter, "Mid-infrared photocurrent measurements on self-assembled Ge dots in Si," Physica E 7, 146-150 (2000).

5. G. J. Hawkins, "Spectral characteristics of infrared optical materials and filters," Ph.D. thesis (University of Reading, 1998).

6. E. D. Palik, Handbook of Optical Constants of Solids (Academic, 1985), vol. 1.

7. R. A. Soref, S. J. Emelett, and W. R. Buchwald, "Silicon waveguided components for the long-wave infrared region," J. Opt. A 8, 840-848 (2006).

8. V. Raghunathan, D. Borlaug, R. R. Rice, and B. Jalali, "Demonstration of a mid-infrared silicon Raman amplifier," Opt. Express 15, 14355-14362 (2007).

9. W. R. Headley, G. T. Reed, M. Pannicia, A. Liu, and S. Howe, "Polarization-independent optical racetrack resonators using rib waveguides in silicon-on-insulator," Appl. Phys. Lett. 85, 5523-5525 (2004).

10. W. N. Ye, D.-X. Xu, S. Janz, P. Cheben, M.-J. Picard, B. Lamontagne, and N. G. Tarr, "Birefringence control using stress engineering in silicon-on-insulator (SOI) waveguides," J. Lightwave Technol. 23, 1308-1318 (2005).

11. K. Kawano and T. Kitoh, Introduction to Optical Waveguide Analysis: Solving Maxwell's Equation and the Schrödinger Equation (Wiley, 2001).

12. R. Scarmozzino, A. Gopinath, R. Pregla, and S. Helfert, "Numerical techniques for modeling guided-wave photonic devices,” IEEE J. Sel. Top. Quantum Electron. 6, 150-162 (2000).

13. "FEMLAB," COMSOL, Inc., Burlington, Mass., www.comsol.com.

14. "Beamprop," Rsoft Design Group, Inc., Ossining, N.Y., www.rsoftdesign.com.

15. K. Okamoto, T. Hosaka, and T. Edahiro, "Stress analysis of optical fibers by a finite element method," IEEE J. Quantum Electron. QE-17, 2123-2129 (1981).
16. S. Timoshenko and J. N. Goodier, Theory of Elasticity, 2nd ed. (McGraw-Hill, 1951).

17. L. D. Landau and E. M. Lifshitz, Theory of Elasticity, 2nd ed. (Pergamon, 1970).

18. M. M. Milošević, P. S. Matavulj, and G. Z. Mashanovich, "Stress-induced characteristics of silicon-on-insulator rib waveguides," in Proceedings 15th Telecommunication Forum-TELFOR (2007), pp. 401-404.

19. R. W. Hoffmann, in Measurement Techniques for Thin Films, B. Schwartz and N. Schwartz, eds. (Electrochemical Society, 1967), p. 312.

20. M. Huang, "Stress effects on the performance of optical waveguides," Int. J. Solids Struct. 40, 1615-1632 (2003).

21. M. Abramowitz and I. A. Stegun, eds., Handbook of Mathematical Functions (Dover, 1970).

22. M. M. Milošević, P. S. Matavulj, and G. Z. Mashanovich, "Interpolation of the zero-birefringent surface by use of Chebyshev polynomial," presented at the 12th Serbian Mathematical Congress, Novi Sad, Serbia, Aug. 28-Sept. 2, 2008.

23. D. Thomson, F. Y. Gardes, G. Z. Mashanovich, A. P. Knights, and G. T. Reed, "Using $\mathrm{SiO}_{2}$ carrier confinement in total internal reflection optical switches to restrict carrier diffusion in the guiding layer," J. Lightwave Technol. 26, 1288-1294 (2008).

24. M. M. Milošević, P. S. Matavulj, B. D. Timotijević, G. T Reed, and G. Z. Mashanovich, "Design rules for singlemode and polarization independent silicon-on-insulator rib waveguides using stress engineering," J. Lightwave Technol. 26, 1840-1846 (2007).

25. G. Z. Mashanovich, M. Milošević, P. Matavulj, S. Stanković, B. Timotijević, P. Y. Yang, E. J. Teo, M. B. H. Breese, A. A. Bettiol and G. T. Reed, "Silicon photonic waveguides for different wavelength regions," Semicond. Sci. Technol. 23, 064002 (2008). 